\title{
Video Article \\ A Method for Quantifying Upper Limb Performance in Daily Life Using Accelerometers
}

\author{
Catherine E. Lang ${ }^{1,2,3}$, Kimberly J. Waddell ${ }^{1}$, Joseph W. Klaesner ${ }^{1,4,5}$, Marghuretta D. Bland ${ }^{1,2,3}$ \\ ${ }^{1}$ Program in Physical Therapy, Washington University School of Medicine \\ ${ }^{2}$ Program in Occupational Therapy, Washington University School of Medicine \\ ${ }^{3}$ Department of Neurology, Washington University School of Medicine \\ ${ }^{4}$ Mallinckrodt Institute of Radiology, Washington University School of Medicine \\ ${ }^{5}$ Department of Biomedical Engineering, Washington University
}

Correspondence to: Catherine E. Lang at langc@wustl.edu

URL: https://www.jove.com/video/55673

DOI: doi: $10.3791 / 55673$

Keywords: Medicine, Issue 122, human movement, hand, arm, measurement, activities of daily living, wearable sensors, outcomes, rehabilitation, accelerometry

Date Published: 4/21/2017

Citation: Lang, C.E., Waddell, K.J., Klaesner, J.W., Bland, M.D. A Method for Quantifying Upper Limb Performance in Daily Life Using Accelerometers. J. Vis. Exp. (122), e55673, doi:10.3791/55673 (2017).

\section{Abstract}

A key reason for referral to rehabilitation services after stroke and other neurological conditions is to improve one's ability to function in daily life. It has become important to measure a person's activities in daily life, and not just measure their capacity for activity in the structured environment of a clinic or laboratory. A wearable sensor that is now enabling measurement of daily movement is the accelerometer. Accelerometers are commercially-available devices resembling large wrist watches that can be worn throughout the day. Data from accelerometers can quantify how the limbs are engaged to perform activities in peoples' homes and communities. This report describes a methodology to collect accelerometry data and turn it into clinically-relevant information. First, data are collected by having the participant wear two accelerometers (one on each wrist) for $24 \mathrm{~h}$ or longer. The accelerometry data are then downloaded and processed to produce four different variables that describe key aspects of upper limb activity in daily life: hours of use, use ratio, magnitude ratio, and the bilateral magnitude. Density plots can be constructed that visually represent the data from the $24 \mathrm{~h}$ wearing period. The variables and their resultant density plots are highly consistent in neurologically-intact, community-dwelling adults. This striking consistency makes them a useful tool for determining if upper limb daily performance is different from normal. This methodology is appropriate for research studies investigating upper limb dysfunction and interventions designed to improve upper limb performance in daily life in people with stroke and other patient populations. Because of its relative simplicity, it may not be long before it is also incorporated in clinical neurorehabilitation practice.

\section{Video Link}

The video component of this article can be found at https://www.jove.com/video/55673/

\section{Introduction}

Over the last two decades, there has been an explosion of interest in wearable sensors to measure movement. A wearable sensor that has generated a great deal of interest in the neurorehabilitation field is the accelerometer. ${ }^{1,2,3}$ Accelerometers, as the name implies, measure accelerations in gravitational units $\left(1 \mathrm{~g}=9.8 \mathrm{~m} / \mathrm{s}^{2}\right)$ or in arbitrary units called activity counts ( 1 activity count = a manufacturer-specified gravitational value). Accelerations, like human movement, are typically measured and recorded in three dimensions, corresponding to the different axes of the device. The devices are commercially available and resemble large wrist watches; they can be worn during daily activities with minimal disruption. Because of the reasonable cost and their ready availability, the use of accelerometers (termed accelerometry) is being integrated into neurorehabilitation research.

The value of accelerometry to the field of neurorehabilitation is that it offers a non-invasive, unbiased, quantitative measure of upper limb motor activity outside the clinic or laboratory. ${ }^{3}$ A key goal of rehabilitation services for people with stroke and other neurological conditions is to improve one's ability to function in daily life, and not just in the clinic or laboratory. The World Health Organization's International Classification of Function distinguishes between the capacity for activity, as measured in a structured environment with clinical tests, and performance of activity, as measured in an unstructured environment. ${ }^{4}$ Accelerometry enables measurement of upper limb performance in the unstructured environment, i.e. what someone actually does when they are not in the clinic or laboratory, not just what they could do. Incorporation of accelerometry into stroke rehabilitation research is now challenging the long-held assumption that functional improvements in a structured clinical environment translate to improvements in performance in unstructured, daily life. ${ }^{5,6,7,8}$

Our group ${ }^{9,10,11,12,13,14}$ and others ${ }^{7,15,16,17,18,19,20,21,22,23,24}$ have been spent a great deal of time and effort on developing accelerometry methodology for use in research and clinical practice. Accelerometry has become well established as a valid and reliable tool for measuring upper limb performance post stroke. ${ }^{1,2,15,16,17,25}$ The most recent challenge has been turning the raw accelerometer data into clinically meaningful 
information (see reference ${ }^{3}$ for a summary of this development process). The methodology described here can be used for distinguishing upper limb performance in daily life in healthy control participants ${ }^{10,12}$ from that in participants who have suffered from stroke $e^{6,9,11}$ or have other disorders. The variables derived from this methodology are responsive to change and quantify improvements over time. ${ }^{14}$ The accelerometer methodology is appropriate for research studies investigating upper limb dysfunction and interventions designed to improve upper limb performance in daily life in people with stroke and other neurologic populations. Because of its relative simplicity, it may not be long before it is also incorporated in clinical neurorehabilitation practice.

\section{Protocol}

This protocol was approved by the Washington University Human Research Protection Office.

NOTE: Instructions were written specific to commercially available accelerometers and their related software for data collection (see Table of Materials).

\section{Preparing the Accelerometers to Collect Data}

1. Connect the two accelerometers to the computer (via docking station) to charge their batteries; this will ensure recording during the entire wearing time.

2. With the accelerometers connected to the computer, open the appropriate software to initialize them.

3. Within the software, select 'Initialization' to synchronize the accelerometer clocks to each other and to the local computer, and set the data collection parameters as follows.

1. Enter (or select from the calendar and clock) the start and end dates and times. Choose the start of data collection based on when the accelerometers will be placed on the participant and an end time at least $24 \mathrm{~h}$ later.

NOTE: The single day wearing period provides a good representation of daily activity in non-employed adults. ${ }^{12}$ Longer wearing periods may be more appropriate for adults or children with varying daily schedules. ${ }^{3,18,26}$

2. Select ' $30 \mathrm{~Hz}$ ' from the dropdown menu for 'Sampling Rate'.

3. Leave 'LED Options' and 'Wireless Options' unchecked.

4. To extend battery life, enable 'Idle Sleep Mode'.

4. To complete the initialization process, select 'Enter Subject Info'.

1. Enter subject specific information for the location of the accelerometer (wrist) and the body side ('Right' or 'Left').

2. Choose to fill in other subject specific information as desired; entry will be for identification only and will not affect the data analyses described here.

3. When ready, select 'Initialize Devices' to complete the process. Once initialization is confirmed, the accelerometers can be safely disconnected from the computer.

\section{Placement and Wearing of the Accelerometers to Collect Data from Participants}

1. Place one accelerometer on each wrist of the participant.

NOTE: The accelerometers should fit snuggly, but not too tightly to the wrist, like a large wrist watch. A variety of bands can be used depending on the size, preference, and comfort level of the participant.

2. Instruct the participant as follows, and answer any questions the participant might have about the wearing period and activity during this time.

1. Ask the participant to do their regular activities throughout the day; the accelerometers may feel strange at first but one soon gets used to them.

2. Instruct them that the accelerometers are waterproof and can be worn while showering or doing the dishes. Instruct them to not wear the accelerometers during extended periods of swimming.

3. Ask them to keep the accelerometers on during naps and overnight.

NOTE: The accelerometers are labeled to identify the left and right sensors. If the accelerometers need to be removed during the wearing period, the labels help identify the correct side when putting them back on. Instruct users to write down on the wearing log when they were taken off and put back on again. The accelerometers are worn at night because, when we let people take them off, they often are not put back on, or are put back on the opposite limbs.

3. Send the participant home with encouragement to engage in normal daily activities and instructions about when to take the two accelerometers off, and how to bring or mail back the accelerometers and wearing log.

\section{Download the Data for Visual Inspection}

1. When the accelerometers have been returned after a wearing period of $24 \mathrm{~h}$ or more, connect the accelerometers to the computer to download the recorded data.

2. Select 'Download' within the appropriate software and then choose a location to store the data on the computer using the 'Change Location' button.

1. Select the option to 'Create AGD file'.

2. For files that are easy to view, choose '10 s' from the 'Epoch' dropdown box. Use these files in Step 3.3.

3. Select 'Download All Devices'.

3. Visually inspect the data to confirm the accelerometers were worn for the planned time period and/or that the data matches the wearing log. 
1. From the top menu, click 'File / Open AGD File' and then select the files to open.

2. Look at the 'Daily Graphs' to see the collected data.

3. Confirm that activity occurred during typical waking hours and that there are not extended periods of no activity, except in the night time. The graphs can be scaled to focus in on smaller increments of time and scrolled through if desired.

\section{Download the Data for Processing}

1. Repeat the Download process (Step 3.2) but this time choose ' $1 \mathrm{~s}$ ' from the 'Epoch' dropdown box. This will bin the data into $1 \mathrm{~s}$ epochs, ${ }^{10,11,12}$ and generate files that will be used for calculations.

NOTE: The accelerometers and software used here (see Table of Materials) use proprietary software to filter out high frequency, non-human activity (e.g. accelerations from being in a car ride). Filtering may need to be done with custom-written software if using different devices and software. Custom-written software can also be used to identify and remove upper limb tremor, such as in a person with Parkinson Disease.

2. From the $1 \mathrm{~s}$ files saved in Step 4.1, calculate a vector magnitude time series of the 3-dimensional data as the square root of $\left(x^{2}+y^{2}+z^{2}\right)$ from data from each accelerometer. This time series can then be used to calculate a number of variables to quantify upper limb activity during daily life.

NOTE: The processing instructions assume a one day wearing period. If the wearing period is longer, data can be processed in separate single day chunks, or as a single time series with calculated variables adjusted by the by the length of the wearing period when appropriate.

\section{Variables and Graphical Representations Created from the Accelerometry Data}

NOTE: Upper limb movements associated with walking are included in the analyzed data. Previous work has established that walking does not influence the accelerometer ratio variables. ${ }^{15}$ Although inclusion of walking does not change the non-ratio variables for neurologically-intact adults, ${ }^{27}$ it is possible that the inclusion of walking could result in a small overestimation of the non-ratio variables for participants with stroke.

1. Calculate hours of use of each limb by summing all the seconds during the recording period when the activity count was non-zero, and then converting to hours. ${ }^{12,17}$

NOTE: This calculation yields one value for each limb.

2. Calculate the use ratio (also called the activity ratio) by dividing the hours of use of the non-dominant limb (or affected limb) by the hours of use of the dominant (or non-affected) limb.

NOTE: The use ratio quantifies the total duration of one limb's activity with respect to the other. ${ }^{12,15}$ This calculation yields a single value, typically between 0 and 1 . A value of 1 indicates the two limbs are used for equal durations throughout the wearing period. A value of zero means that the non-dominant or affected limb was not used at all.

3. Calculate the magnitude ratio as follows.

1. For each second of data in the time series, compute the natural log of the vector magnitude of the non-dominant limb (or affected limb) divided by the vector magnitude of the dominant (or non-affected) limb.

2. Replace values greater than 7 and less than -7 , with 7 and -7 , respectively, to categorize single limb movement. ${ }^{11}$

NOTE: The magnitude ratio quantifies the contribution of each limb to daily activity on a second-by-second basis. ${ }^{10,11}$ This is conceptually similar to the use ratio, but takes into account the intensity of movement (acceleration magnitude) of each limb during each second. This calculation yields a time series of values, where values of zero indicate both limbs had equal movement intensities during that instant in time. Positive values indicate greater movement intensity from the non-dominant (or affected) limb and negative values indicate greater movement intensity from the dominant (or unaffected) limb.

4. Calculate the bilateral magnitude as the sum of the vector magnitude from the two limbs.

NOTE: The bilateral magnitude quantifies the intensity of movement in both upper limbs on a second-by-second basis. ${ }^{10,11}$ This calculation yields a time series of values, where the value indicates the movement intensity, with higher values indicating high intensities.

5. Construct density plots to graphically represent the accelerometry data from both limbs ${ }^{11}$ as follows.

1. Plot each second of data as a bivariate histogram with the frequency represented in color. Set the color scale such that cooler colors (blues) indicate less frequent activity and warmer colors (yellow through red) indicate more frequent activity.

2. Plot the magnitude ratio, indicating the contribution one limb vs. the other, on the x-axis.

3. Plot the bilateral magnitude, indicating the intensity of the movement, on the $y$-axis.

4. Plot the single limb values as separate bars on the far left bar (-7), indicating activity of just the dominant (or unaffected) limb, and on the far right (7), indicating activity of just the non-dominant (or affected) limb.

NOTE: The plots provide a context for scientists, clinicians, and participants to interpret two variables together, the magnitude ratio and the bilateral magnitude. An option to create density plots using accelerometry data is available here. ${ }^{44}$

\section{Representative Results}

Data from a referent sample of community-dwelling, neurologically-intact adults can be used to interpret data from participants with stroke or other conditions affecting upper limb performance. ${ }^{10,11,12}$ Table 1 shows summary statistics for hours of use and the use ratio from a healthy referent sample. Overall, most people are active with their dominant and non-dominant hands for about the same amount of time throughout the day. The average is near $9 \mathrm{~h}$, but there is a wide range, capturing more active and less active people. The average use ratio is just under 1.0 and has a small standard deviation. Thus, regardless of how active one is, the dominant and non-dominant limbs are used for similar durations throughout the day. Further, age does not influence upper limb performance measures in the presence of good health. ${ }^{12}$ Calculated values substantially outside these referent values $( \pm 3-4$ SDs) should be carefully checked to ensure that they are real, as suggested by Uswatte and colleagues. $^{16}$ 


\begin{tabular}{|l|l|l|l|l|}
\hline & Average & Standard Deviation & Minimum & Maximum \\
\hline $\begin{array}{l}\text { Hours of dominant limb } \\
\text { use }\end{array}$ & 9.1 & 1.9 & 4.4 & 14.2 \\
\hline $\begin{array}{l}\text { Hours of non-dominant } \\
\text { limb use }\end{array}$ & 8.6 & 2 & 4.1 & 15.5 \\
\hline Use ratio & 0.95 & 0.06 & 0.79 & 1.1 \\
\hline
\end{tabular}

Table : Summary Accelerometry Statistics from Neurologically-intact, Community Dwelling Adults. Values are from referent sample of 74 community dwelling adults (average age $54 \pm 11,53 \%$ female, $84 \%$ right hand dominant), from reference ${ }^{12}$.

The density plots allow one to take a closer look at the data. Figure 1 is a representative density plot from a healthy adult, with data collected and processed as described above. Plots like this provide important information about upper limb performance in daily life. There are three key features of this plot that are highly consistent across adults of all ages. ${ }^{3,11}$ First, the picture is symmetrical. This indicates that the upper limbs are active together throughout the day, with the dominant and non-dominant limbs used similarly. The similarity of movement may not present be at a specific instance in time, with each limb taking its turn leading or lagging during various activities, but can be seen over the course of the day. Even the bars on either side at -7 and 7 (indicating solely dominant and solely non-dominant activity) are similar in color. The symmetry is contrary to common perceptions about hand dominance. Second, the plot is tree-shaped with a wide bottom portion and rounded edges. The 'rims' or rounded edges of the bottom portion represent activity where one limb is moving while the other is relatively still. An example of this would be placing objects in a container with one hand while holding the container with the other. ${ }^{10}$ The symmetry in the rounded edges indicates that both hands are active to perform and to stabilize similarly over the course of the day. The top peak represents the less frequent, higher intensity activities, such as placing large objects on a high shelf with both hands. ${ }^{10}$ And third, there is a warm glow in the center. This indicates that the most frequent upper limb movements are low intensity with approximately equal contributions from both limbs. Examples of this would be typing or cutting with a knife and fork. ${ }^{10}$

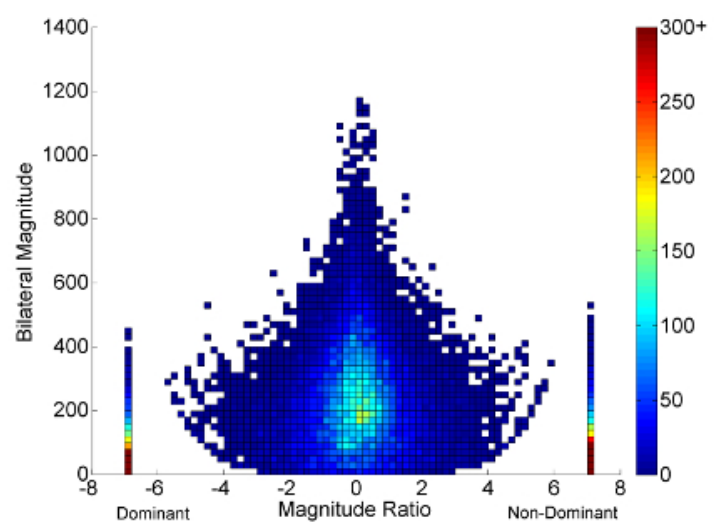

Figure 1: Representative Example from a Neurologically-intact Adult. The density plot shows $24 \mathrm{~h}$ of upper limb use in daily life, plotted on a second-by-second basis. The x-axis (magnitude ratio) indicates the contribution of each limb to activity. The $y$-axis (bilateral magnitude) indicates the intensity of movement. The color represents frequency, with the large color bar scale on the right side of the figure, where brighter colors indicate greater frequencies. The small bars at -7 and 7 represent unilateral dominant and non-dominant activity, respectively. Please click here to view a larger version of this figure.

Across this sample of adults, the density plots are remarkably similar in shape and color. ${ }^{11}$ People who are relatively inactive tend to have shorter, wider, pictures with cooler colors. People who are very active tend to have taller pictures with warmer colors. The striking consistency across adults makes it easy to identify participants with upper limb performance that is different than from these norms.

Figure 2 is an example of a density plot in a person with stroke. This person is a right-handed male who had an ischemic stroke affecting his brain on the right side 11 months prior to these data being collected. The right side of the brain controls the left side of the body, and his left upper limb had moderate paresis and dysfunction, as indicated by a Motricity Index ${ }^{28}$ score of $60 / 100$ and an Action Research Arm Test ${ }^{29}$ score of $38 / 57$. During the $24 \mathrm{~h}$ wearing period, the paretic, left limb was active for $1.5 \mathrm{~h}$ and the non-paretic, right limb was active for $5.8 \mathrm{~h}$. His use ratio was 0.47 , approximately half of the normal value. Compared to the density plot in Figure 1, this density plot is decidedly asymmetrical, indicating that the paretic upper limb was rarely active during daily life. The cool colors of the middle portion of the plot compared to the dark red colors of the single bar at -7 indicate a high frequency of movement with just the non-paretic limb. The overall peak is low, indicating only low intensity activities. Overall, the density plot indicates that the paretic limb participates only minimally in daily activity. 


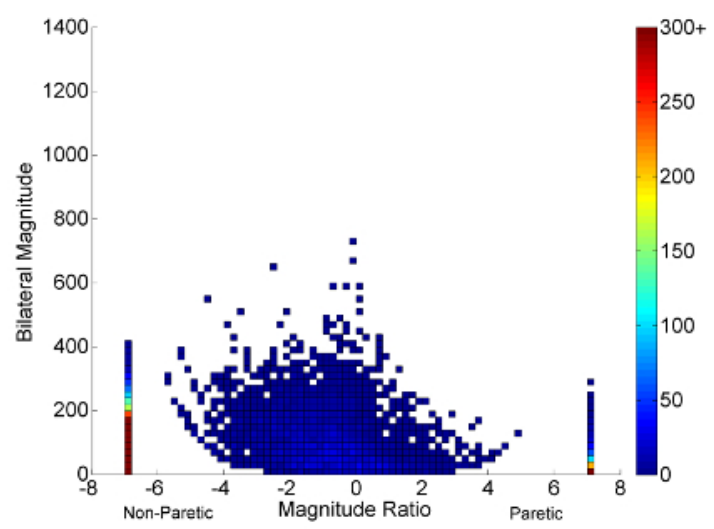

Figure 2: Representative Example from a Person with Stroke. The density plot shows $24 \mathrm{~h}$ of upper limb use in daily life, plotted on a second-by-second basis. The $x$-axis (magnitude ratio) indicates the contribution of each limb to activity. The $y$-axis (bilateral magnitude) indicates the intensity of movement. The color represents frequency, with the large color bar scale on the right side of the figure, where brighter colors indicate greater frequencies. The small bars at -7 and 7 represent unilateral dominant and non-dominant activity, respectively. Compare the symmetry, peak height, and color to Figure 1. Please click here to view a larger version of this figure.

While the accelerometry methodology has been developed for use in persons with stroke, the utility of this methodology extends to other populations. It can be beneficial for evaluating outcomes in a variety of patient populations. Figure 3 is an example of a density plot in a person with an upper limb amputation below the elbow. This individual was a 75 year old male, injured in an accident approximately 8 years ago. His right, previously dominant, hand was amputated at the time of the accident. He owns an upper limb prosthesis but wears it only 1-2 times per month to lift heavy objects. Most of the time, as in this figure, he does not wear it. During the $24 \mathrm{~h}$ wearing period, the intact, left limb was active for $6.9 \mathrm{~h}$ and the residual, right limb was active for $4.7 \mathrm{~h}$ (accelerometer was worn distally on the residual limb). His use ratio was 0.68 , indicating a preference for engaging the intact limb over the residual limb. This density plot is less symmetrical and has cooler colors than that of a control subject (Figure 1), but is more symmetrical and shows more activity than the person with stroke shown in Figure 2. Thus, this person favors the intact limb, but still engages the residual limb in activities during daily life.

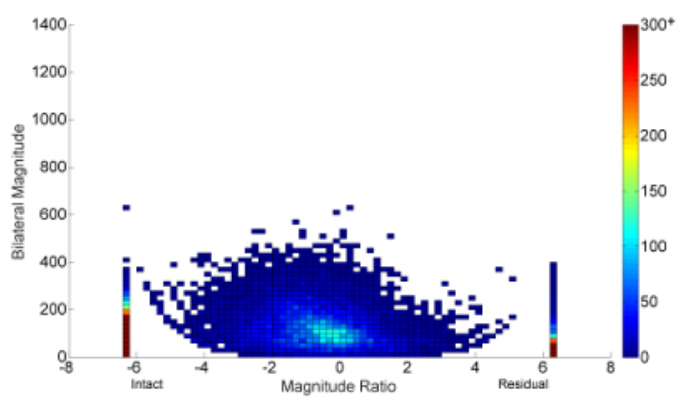

Figure 3: Representative Example from a Person with Upper Limb Amputation. The density plot shows $24 \mathrm{~h}$ of upper limb activity in daily life, plotted on a second-by-second basis. The x-axis (magnitude ratio) indicates the contribution of each limb to activity at the moment in time. The $y$-axis (bilateral magnitude) indicates the intensity of movement. The color represents frequency, with the large color bar scale on the right side of the figure, where brighter colors indicate greater frequencies. The small bars at -7 and 7 represent unilateral dominant and non-dominant activity, respectively. Compare the symmetry, peak height, and color to Figures 1 and 2 . Please click here to view a larger version of this figure.

Another example of how this methodology might be used is in persons with limited mobility who need to increase activity. Figure 4 is an example of a density plot from an elderly, right-handed individual staying in a skilled nursing facility. This person was debilitated after an acute illness and was receiving nursing and rehabilitation services in order to regain independence and return home. The dominant limb was active for 2.4 $\mathrm{h}$ and the non-dominant limb was active for $2.0 \mathrm{~h}$. The use ratio was 0.84 , which is on the low end of the normative range (see Table 1). This density plot is nearly symmetrical, as would be expected from a general medical condition, but the peak is very low and the colors are mostly cool, indicating little activity during the wearing period. 


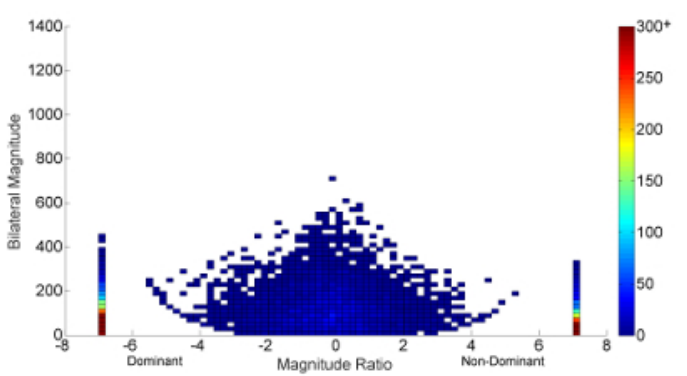

Figure 4: Representative Example from a Person Recovering from Medical Illness in a Skilled Nursing Facility (SNF). The density plot shows $22 \mathrm{~h}$ of upper limb activity in daily life, plotted on a second-by-second basis. The $\mathrm{x}$-axis (magnitude ratio) indicates the contribution of each limb to activity at the moment in time. The y-axis (bilateral magnitude) indicates the intensity of movement. The color represents frequency, with the large color bar scale on the right side of the figure, where brighter colors indicate greater frequencies. The small bars at -7 and 7 represent unilateral dominant and non-dominant activity, respectively. Compare the symmetry, peak height, and color to Figure 1 . Please click here to view a larger version of this figure.

Finally, this methodology may not be just for adults. The protocol is suitable for children, with minor adaptations to encourage wearing (e.g. colorful straps, suggestions that the devices 'make you look like a superhero'). Density plots from typically developing children show the same general shapes as adults, the tree-shape being narrower and the peak substantially higher. The children's shapes are consistent with their greater activity levels; an example of density plots from a typically developing child and a child with hemiparetic cerebral palsy can be seen on p. 25 , Figure $\mathbf{5 B}$ and $\mathbf{5 C}$ in reference ${ }^{3}$. Further investigations are needed for the application to pediatric clinical practice. It is noted that the use ratio has a consistent moderate relationship to self-reporting of upper limb activity in adults with stroke, ${ }^{1}$ but, in children with cerebral palsy, the use ratio is not related to the parent report of upper limb activity. ${ }^{30}$ Whether the altered relationship between sensor-measured and reported values lies in the perceptions of the reporters or in some quantitative or qualitative difference of how children move is unknown. Future studies are sorely needed to determine normative values for typically developing children and investigate the interpretation of values in children with disabilities.

\section{Discussion}

This report details a methodology for measuring upper limb performance in daily life using accelerometers worn on the wrists. Use of this methodology in rehabilitation research and clinical practice affords a significant advancement upon existing methods, i.e. the opportunity to learn how an experimental or typical treatment impacts functional performance in daily life, not just capability in the clinic or laboratory. Accelerometry can be used in conjunction with, or in place of, self-reported measures of daily performance, ${ }^{31,32,33}$ which may be more susceptible to cognitive deficits or unconscious bias. ${ }^{34,35,36,37}$ Early adoption of this methodology has yielded data contrary to expectations, ${ }^{5}$ which could force the field to rethink the content and delivery of rehabilitation services.

Critical steps in the protocol ensure accurate and real data were collected during the wearing period (protocol steps 2.2, 2.3, and 3.3). Failure to follow these steps could result in calculated values that have no meaning. It is relatively easy to make sure that the accelerometers are on the assigned wrists as the person leaves the clinic or laboratory. Visual inspection of the data after the accelerometers are returned is necessary, as participants often behave differently than instructed or anticipated. While relatively rare, participants have been known to remove the accelerometers shortly after leaving the investigative team, putting them back on again on the wrong sides, or trying to encourage others in their family to wear them. Much of this can be avoided if the accelerometers are clearly marked for each side, the wearing log is completed, and the data are inspected shortly after returning, i.e. in case a follow-up telephone call is needed to clarify wearing side and times.

While the accelerometry methodology quantifies general upper limb performance, it does not provide information about movement quality or about specific activities that were performed during the wearing period, such as knowing that a participant was eating; see reference ${ }^{3}$ for a discussion of this issue. As a tool then, accelerometry will be most useful as an outcome measure when the scientific question or rehabilitation intervention is focused on changing general upper limb performance in daily life, such as the amount of activity and the involvement of bilateral limbs in daily activity. Accelerometry will be less useful as an outcome measure when the scientific question or rehabilitation intervention is focused on changing the quality of movement or changing only a few specific movements in daily life. We anticipate that computational methods will improve over time and future generations of this methodology may be able to overcome these limitations.

In conclusion, accelerometry presents an opportunity for quantitative assessment of upper limb performance in daily life. The methodology described here can be considered the upper limb version of the more common mobility methodologies, where steps per day or minutes of moderate physical activity are recorded on wearable devices. ${ }^{38,39,40,41,42,43}$ While developed for persons with stroke, the versatility of the methodology will allow future application in a variety of other populations. Additional methodological development is needed in adult and pediatric neurorehabiliation populations other than stroke to help answer clinical and research questions related to bilateral activity of the upper limbs.

\section{Disclosures}

The authors declare that they have no conflicting financial interests. 


\section{Acknowledgements}

We thank Brittany Hill, Ryan Bailey, and Mike Urbin for their contributions to the accelerometry methodology and data. Funding for this project comes from NIH R01 HD068290.

\section{References}

1. Lang, C. E., Bland, M. D., Bailey, R. R., Schaefer, S. Y., \& Birkenmeier, R. L. Assessment of upper extremity impairment, function, and activity after stroke: foundations for clinical decision making. J Hand Ther. 26 (2), 104-115 (2013).

2. Gebruers, N., Vanroy, C., Truijen, S., Engelborghs, S., \& De Deyn, P. P. Monitoring of physical activity after stroke: a systematic review of accelerometry-based measures. Arch Phys Med Rehabil. 91 (2), 288-297 (2010).

3. Hayward, K. S. et al. Exploring the role of accelerometers in the measurement of real world upper limb use after stroke. Brain Impairment. 17 (1), 16-33 (2016).

4. Towards a common language for Functioning, Disability, and Health: ICF. World Health Organization, Geneva (2002).

5. Waddell, K. J. et al. Does task-specific training improve upper limb performance in daily life post-stroke? Neurorehabil Neural Repair. (2016).

6. Doman, C. A., Waddell, K. J., Bailey, R. R., Moore, J. L., \& Lang, C. E. Changes in Upper-Extremity Functional Capacity and Daily Performance During Outpatient Occupational Therapy for People With Stroke. Am J Occup Ther. 70 (3) (2016).

7. Rand, D., \& Eng, J. J. Predicting daily use of the affected upper extremity 1 year after stroke. J Stroke Cerebrovasc Dis. 24 (2), $274-283$ (2015).

8. Lemmens, R. J. et al. Accelerometry measuring the outcome of robot-supported upper limb training in chronic stroke: a randomized controlled trial. PLoS One. 9 (5), e96414 (2014).

9. Bailey, R. R., Birkenmeier, R. L., \& Lang, C. E. Real-world affected upper limb activity in chronic stroke: an examination of potential modifying factors. Top Stroke Rehabil. 22 (1), 26-33 (2015).

10. Bailey, R. R., Klaesner, J. W., \& Lang, C. E. An accelerometry-based methodology for assessment of real-world bilateral upper extremity activity. PLoS One. 9 (7), e103135 (2014).

11. Bailey, R. R., Klaesner, J. W., \& Lang, C. E. Quantifying Real-World Upper-Limb Activity in Nondisabled Adults and Adults With Chronic Stroke. Neurorehabilitation and Neural Repair. 29 (10), 969-978 (2015).

12. Bailey, R. R., \& Lang, C. E. Upper-limb activity in adults: referent values using accelerometry. J Rehabil Res Dev. 50 (9), $1213-1222$ (2013).

13. Urbin, M. A., Bailey, R. R., \& Lang, C. E. Validity of body-worn sensor acceleration metrics to index upper extremity function in hemiparetic stroke. J Neurol Phys Ther. 39 (2), 111-118 (2015).

14. Urbin, M. A., Waddell, K. J., \& Lang, C. E. Acceleration Metrics Are Responsive to Change in Upper Extremity Function of Stroke Survivors. Arch Phys Med Rehabil. (2014).

15. Uswatte, G. et al. Ambulatory monitoring of arm movement using accelerometry: an objective measure of upper-extremity rehabilitation in persons with chronic stroke. Arch Phys Med Rehabil. 86 (7), 1498-1501 (2005).

16. Uswatte, G. et al. Validity of accelerometry for monitoring real-world arm activity in patients with subacute stroke: evidence from the extremity constraint-induced therapy evaluation trial. Arch Phys Med Rehabil. 87 (10), 1340-1345 (2006).

17. Uswatte, G. et al. Objective measurement of functional upper-extremity movement using accelerometer recordings transformed with a threshold filter. Stroke. 31 (3), 662-667 (2000).

18. Rand, D., Eng, J. J., Tang, P. F., Jeng, J. S., \& Hung, C. How active are people with stroke?: use of accelerometers to assess physical activity. Stroke. 40 (1), 163-168 (2009).

19. Rand, D., Givon, N., Weingarden, H., Nota, A., \& Zeilig, G. Eliciting upper extremity purposeful movements using video games: a comparison with traditional therapy for stroke rehabilitation. Neurorehabil Neural Repair. 28 (8), 733-739 (2014).

20. Connell, L. A., McMahon, N. E., Simpson, L. A., Watkins, C. L., \& Eng, J. J. Investigating measures of intensity during a structured upper limb exercise program in stroke rehabilitation: an exploratory study. Arch Phys Med Rehabil. 95 (12), 2410-2419 (2014).

21. Niet, M., Bussmann, J. B., Ribbers, G. M., \& Stam, H. J. The stroke upper-limb activity monitor: its sensitivity to measure hemiplegic upperlimb activity during daily life. Arch Phys Med Rehabil. 88 (9), 1121-1126 (2007).

22. Vega-Gonzalez, A., Bain, B. J., \& Granat, M. H. Measuring continuous real-world upper-limb activity. Conf Proc IEEE Eng Med Biol Soc. 4 3542-3545 (2005).

23. Vega-Gonzalez, A., \& Granat, M. H. Continuous monitoring of upper-limb activity in a free-living environment. Arch Phys Med Rehabil. 86 (3), 541-548 (2005).

24. Pas, S. C., Verbunt, J. A., Breukelaar, D. E., van Woerden, R., \& Seelen, H. A. Assessment of arm activity using triaxial accelerometry in patients with a stroke. Arch Phys Med Rehabil. 92 (9), 1437-1442 (2011).

25. Lang, C. E., Wagner, J. M., Edwards, D. F., \& Dromerick, A. W. Upper Extremity Use in People with Hemiparesis in the First Few Weeks After Stroke. J Neurol Phys Ther. 31 (2), 56-63 (2007).

26. Rand, D., \& Eng, J. J. Disparity between functional recovery and daily use of the upper and lower extremities during subacute stroke rehabilitation. Neurorehabil Neural Repair. 26 (1), 76-84 (2012).

27. Bailey, R. R. Assessment of Real-World Upper Limb Activity in Adults with Chronic Stroke. Doctoral thesis, Washington University in St. Louis, MO, (2015).

28. Collin, C., \& Wade, D. Assessing motor impairment after stroke: a pilot reliability study. J Neurol Neurosurg Psychiatry. 53 (7), $576-579$ (1990).

29. Yozbatiran, N., Der-Yeghiaian, L., \& Cramer, S. C. A standardized approach to performing the action research arm test. Neurorehabil Neural Repair. 22 (1), 78-90 (2008).

30. Sokal, B., Uswatte, G., Vogtle, L., Byrom, E., \& Barman, J. Everyday movement and use of the arms: Relationship in children with hemiparesis differs from adults. J Pediatr Rehabil Med. 8 (3), 197-206 (2015).

31. Uswatte, G., Taub, E., Morris, D., Light, K., \& Thompson, P. A. The Motor Activity Log-28: assessing daily use of the hemiparetic arm after stroke. Neurology. 67 (7), 1189-1194 (2006). 
32. Duncan, P. W. et al. The stroke impact scale version 2.0. Evaluation of reliability, validity, and sensitivity to change. Stroke. $\mathbf{3 0}$ (10), 2131-2140 (1999).

33. Simpson, L. A., Eng, J. J., Backman, C. L., \& Miller, W. C. Rating of Everyday Arm-Use in the Community and Home (REACH) scale for capturing affected arm-use after stroke: development, reliability, and validity. PLoS One. 8 (12), e83405 (2013).

34. Bradburn, N. M., Rips, L. J., \& Shevell, S. K. Answering autobiographical questions: the impact of memory and inference on surveys. Science. 236 (4798), 157-161 (1987).

35. Tatemichi, T. K. et al. Cognitive impairment after stroke: frequency, patterns, and relationship to functional abilities. $J$ Neurol Neurosurg Psychiatry. 57 (2), 202-207 (1994).

36. Adams, S. A. et al. The effect of social desirability and social approval on self-reports of physical activity. Am J Epidemiol. 161 (4), $389-398$ (2005).

37. Prince, S. A. et al. A comparison of direct versus self-report measures for assessing physical activity in adults: a systematic review. Int $J$ Behav Nutr Phys Act. 556 (2008).

38. Cavanaugh, J. T. et al. Capturing ambulatory activity decline in Parkinson's disease. J Neurol Phys Ther. 36 (2), $51-57$ (2012).

39. Paul, S. S. et al. Obtaining Reliable Estimates of Ambulatory Physical Activity in People with Parkinson's Disease. J Parkinsons Dis. (2016).

40. Danks, K. A., Roos, M. A., McCoy, D., \& Reisman, D. S. A step activity monitoring program improves real world walking activity post stroke. Disabil Rehabil. 36 (26), 2233-2236 (2014).

41. Roos, M. A., Rudolph, K. S., \& Reisman, D. S. The structure of walking activity in people after stroke compared with older adults without disability: a cross-sectional study. Phys Ther. 92 (9), 1141-1147 (2012).

42. Mudge, S., \& Stott, N. S. Test--retest reliability of the StepWatch Activity Monitor outputs in individuals with chronic stroke. Clin Rehabil. 22 (10-11), 871-877 (2008).

43. Mudge, S., Stott, N. S., \& Walt, S. E. Criterion validity of the StepWatch Activity Monitor as a measure of walking activity in patients after stroke. Arch Phys Med Rehabil. 88 (12), 1710-1715 (2007).

44. Accelerometry - Program in Physical Therapy. | Program in Physical Therapy. https://accelerometerchart.wusm.wustl.edu/ (2016). 